\title{
Immunolocalization of Gas7 in the Subgranular Zone of Mice Hippocampus
}

\section{Udaya Kumar}

Unit of Biochemistry, Department of Zoology, University of Madras, Chennai, Tamil Nadu, India

Received July 17, 2019; Accepted December 1, 2019.

Key words: Gas7 - Neural progenitor cell - Differentiation - Subgranular zone

Abstract: It is established that neural stem cells (NSC) reside in the hippocampal subgranular zone (SGZ) and contribute to neurogenesis throughout life. Although the molecular events that regulate hippocampal neural stem cell differentiation are known, the precise mechanism is undefined. Here, the role of growth arrest specific 7 (Gas7) protein in SGZ neural progenitor cell differentiation was studied. Immunoblotting and immunohistochemistry for Gas7 were performed on 12 weeks old mice hippocampus. The results displayed a strong signal for Gas7 protein in the mice hippocampal tissue and SGZ cells, therefore suggesting that Gas7 might have a role in neural progenitor cell differentiation in SGZ.

Mailing Address: Udaya Kumar, MSc., PhD., Department of Neurology, University of California Los Angeles, 710 Westwood Plaza, Suite 2253, Los Angeles, California 90095, USA; Phone: +1 (309) 53388 33; e-mail: udevaraj@mednet.ucla.edu 


\section{Introduction}

Growth arrest-specific genes (Gas) were first discovered in growth arrested cultured cells (Schneider et al., 1988). Individual Gas gene is shown to involve in a variety of biological process that regulates apoptosis (Fabbretti et al., 1995), cytoskeleton arrangement (Brancolini et al., 1992), receptor tyrosine kinase activity (Del Sal et al., 1994), cell cycle control (Li et al., 1996), and nerve cell growth (Adlkofer et al., 1995). Studies from our laboratory and other laboratories have demonstrated that one of the Gas genes, Gas7 have a specialized role in neuronal development (Ju et al., 1998; Moorthy et al., 2005). Reports suggest that Gas7 plays an important role in neuritogenesis and neuronal migration (Chao et al., 2003). In 1998, Ju et al. have reported that inhibiting Gas7 gene prevents neurite outgrowth in Purkinje cells. Conversely, over-expression of Gas7 has been shown to promote neurite extension in Neuro2A and PC12 cells (Chao et al., 2003).

Neurogenesis is an important mechanism for brain development (Pan et al., 2013). In the hippocampus, the subgranular zone (SGZ) continuously produces new neurons (Kumar and Devaraj, 2012). In the SGZ, neural stem cells (NSC) proliferate into progenitor cells (Warner-Schmidt and Duman, 2006). The progenitor cells differentiate into neurons and integrate into the hippocampal circuitry (WarnerSchmidt and Duman, 2006; Kumar et al., 2012). Hippocampal neurogenesis involves in memory formation and plays a critical role in mood regulation (Warner-Schmidt and Duman, 2006).

Though, there is progress in characterizing the neurotransmitters and trophic factors that regulate neural progenitor cell differentiation in SGZ (Gage, 2000; Hagg, 2009) the precise mechanism is yet to be defined. Much less is known about the intracellular cascades that influence neural progenitor cell differentiation in SGZ. Improved comprehension of the mechanisms involved in neural progenitor cell differentiation is thus of major importance for brain repair. In this study, the role of Gas7 in neural progenitor cell differentiation was investigated. The data suggests that Gas7 may be an important determinant for neural progenitor cell differentiation in SGZ.

\section{Material and Methods}

Animals

This study consisted of twelve male Swiss albino mice (12 weeks old), where six mice were used for immunoblotting and remaining for immunohistochemistry. They were obtained from Kings Institute of Preventive Medicine, Chennai, India. Mice were housed on a 12:12 hour light-dark cycle with free access to food and water. All procedures were performed in accordance to the laws and conditions of The Committee for the Purpose of Control and Supervision of Experiments on Animals in India (CPCSEA) and Institutional Animal Ethics Committee (IAEC) guidelines. 


\section{Immunohistochemistry}

Mice were deeply anesthetized by intraperitoneal injection of sodium pentobarbital $(100 \mathrm{mg} / \mathrm{kg})$ and perfused through the ascending aorta for 30 minutes with cold saline and $4 \%$ paraformaldehyde. Brains were dissected and stored in the same fixative overnight at $4^{\circ} \mathrm{C} .8 \mu \mathrm{m}$ thick coronal sections containing dorsal hippocampal formation were dewaxed in xylene and rehydrated in a series of ethanol solution. Endogenous peroxidase activity was blocked using $3 \%$ hydrogen peroxide and non-specific binding was blocked with $3 \%$ bovine serum albumin in phosphate-buffered saline for 60 minutes. The sections were incubated overnight at $4{ }^{\circ} \mathrm{C}$ with the Gas7 antibody (dilution 1:200, rabbit anti-Gas7 antiserum was generated against histidine-tagged full-length Gas7 protein expressed in Escherichia coli from plasmid pET15b, a kind gift from Dr. Sue Lin-Chao, Academia Sinica, Taiwan). Slides were washed with phosphate-buffered saline and incubated with horse anti-rabbit biotinylated secondary antibody (BA-1100, Vector Labs) for 2 hours at room temperature. They were developed with 3,3'-diaminobenzidine solution and were visualized using Axioskop-2 Plus Phase Contrast Microscope (Carl Zeiss). Negative controls included substituting the primary antibody with $3 \%$ bovine serum albumin, which resulted in negative staining.

\section{Immunoblot}

Fresh hippocampal tissues were homogenized by an ultrasonic disruption in icecold RIPA buffer (R0278, Sigma-Aldrich) containing protease and phosphatase inhibitors (5892970001 and 4906845001, Roche). After centrifugation, the protein level in the supernatant was determined using Micro BCA protein assay kit (23235, Thermo Scientific) with bovine serum albumin as the standard. The proteins were separated by sodium dodecyl sulphate-polyacrylamide gel electrophoresis and were electrophoretically transferred to PVDF membrane (10600023, Amersham Biosciences). The membranes were stained with Ponceau $\mathrm{S}$ solution to evaluate the protein transfer. They were later blocked in $5 \%$ milk-Tris-buffered saline-Tween and were incubated with Gas7 (dilution 1:1000) and corresponding secondary antibody. The immunoblots were developed using ECL solution and chemiluminescentcompatible film (RPN2232 and 28906835, Amersham Biosciences).

\section{Results}

Detection of Gas7 in the hippocampal tissue Immunoblot analysis of extracts prepared from 12 weeks old mice hippocampus showed positive immunoreactivity for Gas7 in the above stage. Expression of $48 \mathrm{kDa}$ Gas7 protein indicates that Gas7 is physiologically present in the hippocampal tissue (Figure 1).

Localization of Gas7 in the subgranular zone cells Immunohistochemical analysis of mice hippocampal dentate gyrus showed a high degree of positive immunostaining for Gas7 in the stem cell-rich subgranular zone. 




Figure 1 - Immunoblot analysis of mice hippocampal tissue lysate. A single 48 kDa Gas7 protein band was observed.

The signal for Gas7 was prominent in the cytoplasm of subgranular zone cells that indicates Gas7 has a vital role in these cells. A few cells in the granular cell layer also displayed immunoreactivity for Gas7, but their function in granular cells is unclear at this stage (Figure 2).

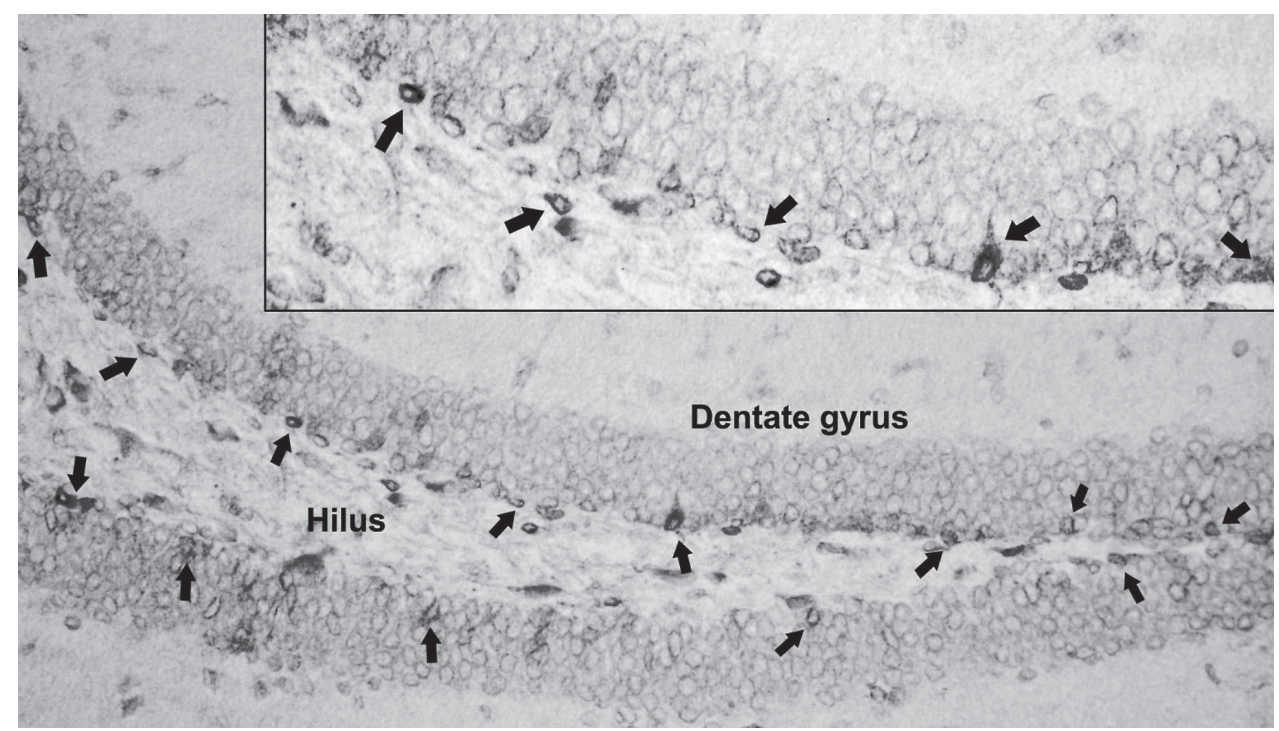

Figure 2 - Immunohistochemical staining of Gas7 in the mice hippocampal dentate gyrus. Gas 7 is present in the SGZ of the dentate gyrus (arrow). Upper inset: magnified view of the dentate gyrus showing strong cytosolic localization of Gas7 in the SGZ cells (arrows). 


\section{Discussion}

Gas7 expresses in various regions of the brain such as cerebral cortex, cerebellum, hippocampus and plays critical role in brain development (Ju et al., 1998). In particular, Gas7 induces neurite outgrowth. Report by Hung et al. (2011) shows that Gas7 regulates mesenchymal stem cell differentiation. This implies that Gas7 has a role in cell differentiation process. In the previous report, our laboratory has proved that Gas7 facilitates embryonic cell mobility via cytoskeletal reorganization (Moorthy et al., 2005), which shows that Gas7 could interact with cytoskeleton to promote cell migration. These facts indicate that Gas7 might help in cell differentiation by modulating the cytoskeletons.

Numerous studies have shown that cytoskeletons play a major role in cell differentiation (Yourek et al., 2007). Reports suggest actin and microtubules form a protein network in the cell cytoplasm and generate a force that makes cell to differentiate. Disrupting this cytoskeletal network would weaken the cell membrane elasticity, leading to failure of cell differentiation (Fletcher and Mullins, 2010). Previous studies on Gas7 shows that Gas7 interacts with actin and microtubules. It plays a crucial role in actin assembly and microtubule stabilization (She et al., 2002; Uchida et al., 2009). In the present study, a strong signal for Gas7 protein in the hippocampal tissue lysate was noticed. Immunohistochemistry data confirms Gas7 accumulation in the cytoplasm of SGZ cells. The localization of the Gas7 protein in the hippocampal neurogenic niche suggests that Gas7 might have a role in SGZ neural progenitor cell differentiation. The precise mechanism of SGZ progenitor cell differentiation by Gas7 is unclear at this stage. However, one mechanism where Gas7 might aid in cell differentiation is through cytoskeletal reorganization. Gas7 may help to form stress fibers in the cell cytoplasm by cross-linking actin

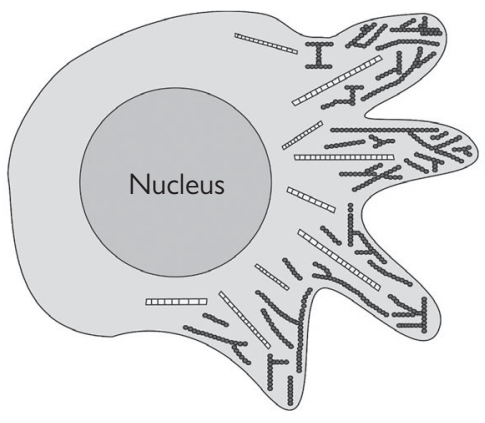

Cell Differentiation

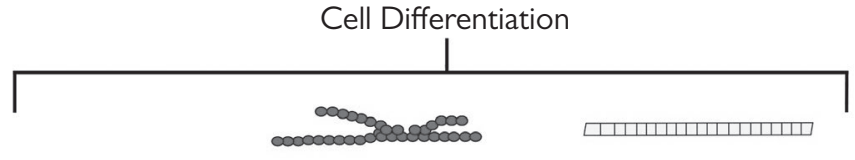

Gas7 Cross-Link F-Actin
+ Polymerize Microtubule
Figure 3 - Schematic representation of cytoskeleton mediated neural progenitor cell differentiation. Gas7 crosslinks F-actin and aids in the polymerization of microtubules. This process within the cell encourages to change its morphology and differentiate. 


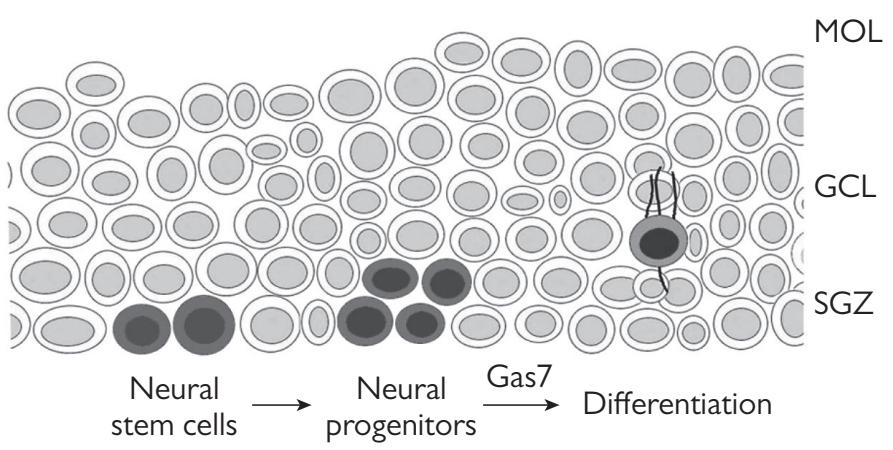

Figure 4 - Schematic representation of neural progenitor cell differentiation in the hippocampal dentate gyrus. The neural stem cells in the SGZ proliferate into neural progenitor cells. The progenitor cells, with the help of Gas7 differentiate into neurons, which may later integrate into the hippocampal circuitry.

filaments and polymerizing microtubules. The stress developed due to the change in cytoskeletal structure could induce the cell to change its morphology, leading to cell differentiation (Figures 3 and 4). Further investigation on Gas7 is needed to resolve the mechanism of Gas7 mediated SGZ neural progenitor cell differentiation. Understanding the functional role of SGZ neural progenitor cell differentiation as well as its underlying cellular and molecular substrates will enable us to identify potential interventions with hippocampal diseases and develop targeted therapies for memory-related disorders.

Acknowledgements: The author thanks Divya Chandrababu and Vahin Uday for their support.

\section{References}

Adlkofer, K., Martini, R., Aguzzi, A., Zielasek, J., Toyka, K. V., Suter, U. (1995) Hypermyelination and demyelinating peripheral neuropathy in Pmp22-deficient mice. Nat. Genet. 11, 274-280.

Brancolini, C., Bottega, S., Schneider, C. (1992) Gas2, a growth arrest-specific protein, is a component of the microfilament network system. J. Cell Biol. 117, 1251-1261.

Chao, C. C., Su, L. J., Sun, N. K., Ju, Y. T., Lih, J. C., Chao, S. L. (2003) Involvement of Gas7 in nerve growth factor-independent and dependent cell processes in PC12 cells. J. Neurosci. Res. 74, $248-254$.

Del Sal, G., Collavin, L., Ruaro, M. E., Edomi, P., Saccone, S., Valle, G. D., Schneider, C. (1994) Structure, function, and chromosome mapping of the growth-suppressing human homologue of the murine Gas 1 gene. Proc. Natl. Acad. Sci. U. S. A. 91, 1848-1852.

Fabbretti, E., Edomi, P., Brancolini, C., Schneider, C. (1995) Apoptotic phenotype induced by overexpression of wild-type Gas3/PMP22: Its relation to the demyelinating peripheral neuropathy CMT1A. Genes Dev. 9 , 1846-1856.

Fletcher, D. A., Mullins, R. D. (2010) Cell mechanics and the cytoskeleton. Nature 463, 485-492.

Gage, F. H. (2000) Mammalian neural stem cells. Science 287, 1433-1438.

Hagg, T. (2009) From neurotransmitters to neurotrophic factors to neurogenesis. Neuroscientist 15, $20-27$.

Hung, F. C., Chang, Y., Chao, S. L., Chao, C. C. (2011) Gas7 mediates the differentiation of human bone marrow-derived mesenchymal stem cells into functional osteoblasts by enhancing Runx2-dependent gene expression. J. Orthop. Res. 29, 1528-1535. 
Ju, Y. T., Chang, A. C., She, B. R., Tsaur, M. L., Hwang, H. M., Chao, C. C., Cohen, S. N., Chao, S. L. (1998) Gas7: A gene expressed preferentially in growth-arrested fibroblasts and terminally differentiated Purkinje neurons affects neurite formation. Proc. Natl. Acad. Sci. U. S. A. 95, 11423-11428.

Kumar, D. U., Devaraj, H. (2012) Expression of Wnt 3a, $\beta$-catenin, cyclin D1 and PCNA in mouse dentate gyrus subgranular zone (SGZ): A possible role of Wnt pathway in SGZ neural stem cell proliferation. Folia Biol. (Praha) 58, 115-120.

Kumar, D. U., Nagaraj, R., Devaraj, H. (2012) Immunolocalization of Notch1, Hes1 and NF-kB in the murine hippocampal subgranular zone (SGZ): Possible role of the Notch pathway in the maintenance of the SGZ neural stem cell population. Neurophysiology 44, 208-215.

Li, R., Chen, J., Hammonds, G., Phillips, H., Armanini, M., Wood, P., Bunge, R., Godowski, P. J., Sliwkowski, M. X., Mather, J. P. (1996) Identification of Gas6 as a growth factor for human Schwann cells. J. Neurosci. 16, 2012-2019.

Moorthy, P. P., Kumar, A. A., Devaraj, H. (2005) Expression of the Gas7 gene and Oct4 in embryonic stem cells of mice. Stem Cells Dev. 14, 664-670.

Pan, Y. W., Wang, W., Xia, Z. (2013) Assessment of adult neurogenesis in mice. Curr. Protoc. Toxicol. 12, 12-20.

Schneider, C., King, R. M., Philipson, L. (1988) Genes specifically expressed at growth arrest of mammalian cells. Cell 54, 787-793.

She, B. R., Liou, G. G., Chao, S. L. (2002) Association of the growth-arrest-specific protein Gas7 with F-actin induces reorganization of microfilaments and promotes membrane outgrowth. Exp. Cell Res. 273, 34-44.

Uchida, T., Akiyama, H., Sakamoto, W., Koga, T., Yan, K., Uchida, C., Hirose, K., Itoh, T. J. (2009) Direct optical microscopic observation of the microtubule polymerization intermediate sheet structure in the presence of Gas7. J. Mol. Biol. 391, 849-857.

Warner-Schmidt, J. L., Duman, R. S. (2006) Hippocampal neurogenesis: Opposing effects of stress and antidepressant treatment. Hippocampus 16, 239-249.

Yourek, G., Hussain, M. A., Mao, J. J. (2007) Cytoskeletal changes of mesenchymal stem cells during differentiation. ASAIO J. 53, 219-228. 